\title{
The Sensitivity of Estimates of Multiphase Fluid and Solid Properties of Porous Rocks to Image Processing
}

\author{
Gaetano Garfi $^{1}$ (D) . Cédric M. John ${ }^{1}$. Steffen Berg ${ }^{1,2,3} \cdot$ Samuel Krevor $^{1}$
}

Received: 26 June 2019 / Accepted: 27 November 2019 / Published online: 7 December 2019

(c) The Author(s) 2019

\begin{abstract}
X-ray microcomputed tomography (X-ray $\mu$-CT) is a rapidly advancing technology that has been successfully employed to study flow phenomena in porous media. It offers an alternative approach to core scale experiments for the estimation of traditional petrophysical properties such as porosity and single-phase flow permeability. It can also be used to investigate properties that control multiphase flow such as rock wettability or mineral topology. In most applications, analyses are performed on segmented images obtained employing a specific processing pipeline on the greyscale images. The workflow leading to a segmented image is not straightforward or unique and, for most of the properties of interest, a ground truth is not available. For this reason, it is crucial to understand how image processing choices control properties estimation. In this work, we assess the sensitivity of porosity, permeability, specific surface area, in situ contact angle measurements, fluid-fluid interfacial curvature measurements and mineral composition to processing choices. We compare the results obtained upon the employment of two processing pipelines: non-local means filtering followed by watershed segmentation; segmentation by a manually trained random forest classifier. Single-phase flow permeability, in situ contact angle measurements and mineral-to-pore total surface area are the most sensitive properties, as a result of the sensitivity to processing of the phase boundary identification task. Porosity, interfacial fluidfluid curvature and specific mineral descriptors are robust to processing. The sensitivity of the property estimates increases with the complexity of its definition and its relationship to boundary shape.
\end{abstract}

Keywords X-ray imaging · Image processing · Contact angle · Fluid-fluid curvature · Mineralogy

Gaetano Garfi

g.garfi17@imperial.ac.uk

1 Department of Earth Science and Engineering, Imperial College London, London, UK

2 Shell Global Solutions International B.V., Amsterdam, The Netherlands

3 Department of Chemical Engineering, Imperial College London, London, UK 


\section{Introduction}

The investigation of multiphase flow phenomena in permeable media is of key interest in several research fields including hydrocarbon recovery (Blunt 2017), carbon sequestration (Krevor et al. 2015), catalyst design (Wu et al. 2005; Lee et al. 2008), fuel cells (Debe 2012; Andersson et al. 2016) and battery efficiency improvement (Newman 1995; Sikha et al. 2004). There has long been interest in mechanisms underlying physical and chemical processes happening at the smallest characteristic length scales controlling flow and transport, the pores and fluid-solid interfacial areas within a system. However, their understanding has been limited by difficulties in observing key fluid and transport properties at the small scales of interest, within the opaque porous media.

In this context, the development of laboratory-based X-ray microcomputed tomography (X-ray $\mu$-CT) has led to rapid advances (Wildenschild and Sheppard 2013). In recent years, $\mathrm{X}$-ray $\mu$-CT has been used in the characterization of rock and fluid flow properties (Blunt et al. 2013; Bultreys et al. 2016a, b). This has extended beyond estimates of simple rock and single-phase flow properties (pore volume, single-phase flow permeability) to include rock mineral composition (Lai et al. 2015), capillary pressure from fluid-fluid interfacial curvature (Armstrong et al. 2012; Lin et al. 2018), and the wetting state from fluid interfacial curvature and in situ contact angle measurements (AlRatrout et al. 2018; Andrew et al. 2014a; Scanziani et al. 2017; AlRatrout et al. 2017).

Imaging and image processing are central to the quality of these observations (Schlüter et al. 2014; Iassonov et al. 2009). Rocks are imaged while saturated or partially saturated by one or more fluids, e.g. air, water, oil, $\mathrm{CO}_{2}$. The 3D image is a map of greyscale values proportional to a linear attenuation coefficient codified as a floating point or an integer number in 16 bits, where the constitutive element is a voxel (three-dimensional pixel). Quantitative information is derived from the image by assigning a phase to each voxel through a classification procedure called image segmentation. The segmentation procedure is often preceded by filtering. The workflow consisting of artefact removal, reconstruction, filtering and segmentation constitutes the image processing task. This is thus the task which defines and builds the $3 \mathrm{D}$ dataset on which measurements are performed and simulations are run. Many alternatives are available for any of the steps constituting the image processing task. The ideal way to select the best processing pipeline consists in testing different variations on synthetic images of the same kind as the one image of interest (Berg et al. 2018; Andrew 2018).

The sensitivities of the measurement to the image processing depend on both the processing pipeline used and the observation of interest. The sensitivities have been evaluated for porosity, single-phase flow permeability and fluid-fluid interfacial curvature (Saxena et al. 2017; Leu et al. 2014; Armstrong et al. 2012). A leading challenge in evaluating image processing is the acquisition of an independent ground truth measurement of the property of interest for benchmarking. Laboratory measured porosity and permeability have been used. However, it is challenging to reconcile porosity estimates from $\mu$-CT images with those that are experimentally determined by standard laboratory methods. There are a number of causes for this including the presence of sub-resolution porosity especially in carbonates or clay minerals (Sok et al. 2010; Andrew et al. 2013; Soulaine et al. 2016). Leu et al. (2014) have also found it difficult to match at the same time porosity and permeability laboratory measurements with estimates from $\mu$-CT images. In general, transport property estimates have also been found sensitive to processing choices (Leu et al. 2014; Saxena et al. 2017). The challenge is accentuated for the observation of multiphase flow 
characteristics for which there are few or no practical independent measurements available other than X-ray CT. As a result, it is key to understand these sensitivities to imaging, processing and analysis protocols.

The aim of this work is to investigate the role of the image processing pipeline choice for the measurement of multiphase flow properties beyond simple binary segmentation of the rock medium and estimation of single-phase flow properties. Thus, we evaluate the sensitivity of porosity and single-phase flow permeability to image processing as a benchmark. We subsequently focus our evaluation on specific surface area (interfacial area between the pore space and the rock matrix, divided by rock matrix volume), local threephase contact angles, mean fluid-fluid interfacial curvature, and mineral volume fraction on five rock samples with distinct pore structures. The analysis of most of these properties requires multiclass segmentation, more challenging than standard binary segmentation. We compared the results obtained from the employment of two processing pipelines. The more widely used pipeline consisted of the sequential use of non-local means filtering (Buades et al. 2005) and watershed segmentation (Beucher and Meyer 1993). The second pipeline consisted in a machine learning based segmentation tool that eliminates the need of a filtering step, Trainable WEKA Segmentation 3D (TWS) (Arganda-Carreras et al. 2017).

\section{Data and Methods}

\subsection{Datasets}

Five rock samples were considered in this study: Bentheimer sandstone, Berea sandstone, Ketton limestone, Edwards limestone and Estaillades limestone. For reference, previously measured laboratory mineral compositions are reported in Table 1 from Lai et al. (2015) and Peksa et al. (2015). We imaged five cylindrical rock samples with diameters of $4 \mathrm{~mm}$ with an FEI Heliscan microCT. The source voltage and the tube current were $75 \mathrm{kV}$ and $95 \mathrm{~mA}$, respectively. The attenuated radiation was measured with a flat panel detector of $2800 \times 2800$ pixels. The $2 \mathrm{D}$ raw images acquired through a helical trajectory were then reconstructed employing an iterative back projection algorithm, provided by the instrument

Table 1 Example mineral composition (weight percentage) from literature XRD/XRF measurements of the five rock lithologies considered (Lai et al. 2015; Peksa et al. 2015)

\begin{tabular}{llcccc}
\hline & Bentheimer & Berea & Ketton & Edwards & Estaillades \\
\hline Dol & 0.26 & $0.2-0.4$ & 0 & 45.4 & 0 \\
Ank & 0 & $0.7-1.3$ & 0 & 0 & 0 \\
Cal & 0.15 & $1.3-2.9$ & 98.3 & 47 & 99.6 \\
Qtz & 91.7 & $70.3-73.1$ & 1.7 & 7.6 & 0.4 \\
Or & 4.86 & $13.1-12.6$ & 0 & 0 & 0 \\
Ab & 0 & 1.0 & 0 & 0 & 0 \\
An & 0 & $1.5-1.7$ & 0 & 0 & 0 \\
Kln & 2.5 & $1.6-2.1$ & 0 & 0 & 0 \\
Ill & 0 & $1.7-2.4$ & 0 & 0 & 0 \\
Sme & 0 & $1.9-2.3$ & 0 & 0 & 0 \\
Chm & 0 & $0.7-1.0$ & 0 & 0 & 0 \\
Clc & 0 & $0.3-0.5$ & 0 & 0 & 0 \\
Py & 0.03 & $1.7-2.6$ & 0 & 0 & 0 \\
\hline
\end{tabular}


manufacturer. The voxel size obtained was $2.5 \mu \mathrm{m}$. The analysis of the role of image processing in estimating rock properties was performed on five subvolumes $\left(400^{3}\right.$ voxels, i.e. $1 \mathrm{~mm}^{3}$ ) extracted from the tomograms of the five rocks. The analysis of the sensitivity of mineral topological characterization was only conducted on a subvolume $\left(500^{3}\right.$ voxels, i.e. $1.95 \mathrm{~mm}^{3}$ ) extracted from the Berea sandstone because it was the only sample with significant mineralogical heterogeneity. The analysis was conducted on subvolumes due to the computational requirements of Trainable WEKA Segmentation 3D (TWS).

In order to evaluate the sensitivity of the estimates of contact angle and interfacial fluid-fluid curvature, a publicly available image of a trapped decane ganglion from Singh and Blunt (2018) was considered. The image was acquired during a drainage-waterflooding experiment in a water-wet Ketton limestone sample. Image voxel size was $2 \mu \mathrm{m}$. A detailed description of the dataset and the protocol of the experiment from which it was obtained is provided in Scanziani et al. (2017).

\subsection{Image Processing Methods}

\subsubsection{Image Processing Pipeline 1: Non-local Means Filtering and Watershed Segmentation}

The first image processing pipeline used makes use of a filter and segmentation combination that has been widely used in studies of porous rocks. Filtering options typically applied in imaging permeable media are reviewed in Kaestner et al. (2008). While computationally cheap filters such as the median and Gaussian filters typically exhibit good performance in reducing white noise and greyscale value outliers, they weaken the contrast in the edges between phases. In order to preserve these edges, more sophisticated filtering algorithms have been developed, such as anisotropic diffusion or Bayesian information theoretic techniques (Wildenschild and Sheppard 2013). Among the many alternatives, the non-local means filtering algorithm has proven to be able to suppress image noise without significantly altering the information content of phase boundaries of an image (Buades et al. 2005; Schlüter et al. 2014). As a consequence, non-local means algorithms have been the preferred filtering choice in many studies in the context of digital rock technology (Singh et al. 2016; Alhammadi et al. 2017; Scanziani et al. 2018). For this reason, a non-local means algorithm was chosen as the filtering option of interest in this study. The implementation available in Thermofisher Avizo Fire 9.5 was employed, choosing a similarity value of 0.3 . This value represents a weighting factor assigned to each voxel inside the search window during the smoothing procedure. The larger the similarity value, the more the image will be smoothed. A value of 0.3 was chosen to avoid oversmoothing, and the results of the filtering process were evaluated by visual inspection. In general terms, the higher the signal-to-noise ratio in the original greyscale image, the lower is the value of the smoothing extent to be chosen.

A review of some of the most common segmentation algorithms can be found in Iassonov et al. (2009). In the past, algorithms based on a global thresholding principle, either manual or automatic, were the most widely used segmentation approaches. Iassonov et al. (2009) have found their performances inferior to algorithms accounting for spatially varying image information. Analogously, Schlüter et al. (2014) identified Bayesian Markov random field segmentation, watershed segmentation and converging active contour segmentation to perform better in multiclass classification. As a consequence, in recent years, the favoured algorithms are those involving the use of spatially specific statistics such as the 
gradient in voxel greyscale intensity. However, most of these algorithms are biased by the requirement of subjective user inputs (Wildenschild and Sheppard 2013). Leu et al. (2014) quantified the sensitivity to the user choice of input parameters of three segmentation algorithms, i.e. global thresholding, hysteresis thresholding and watershed. The use of the latter led to the most robust estimates of porosity, permeability and capillary pressure values in Berea sandstone.

Considering the positive results obtained from the employment of watershed segmentation and the diffusion of this segmentation algorithm in the community (Andrew et al. 2014b; Lin et al. 2018; Alhosani et al. 2019), we chose to employ the watershed algorithm to segment images filtered by non-local means filtering. The watershed algorithm transforms the greyscale values of each pixel or voxel in a greyscale image into a magnitude, sometimes referred to as a height value (Beucher and Meyer 1993). Boundaries and seeds of the phases to segment are identified as the regions where the gradient is at a maximum or a minimum. The labels for the phases then expand from previously identified seeds in a way analogous to water filling crevices in a flooding process. In this study, watershed segmentation was performed using Thermofisher Avizo Fire 9.5 software. In the chosen implementation, the user selects the number of the phases to segment, the gradient magnitude defining phase boundaries and the threshold values that define seeds for each of the phases selected. These choices are not automated and depend on user preference. Therefore, different segmented images would result from different user choices in selecting watershed algorithm's parameters.

\subsubsection{Image Processing Pipeline 2: Machine Learning Based Segmentation}

Recently, the research community has shown interest in testing machine learning algorithms to segment porous media CT images. Cortina-Januchs et al. (2011) employed three unsupervised clustering algorithms, K-means, fuzzy-C-means and self-organizing maps, to build the feature vector used to train an artificial neural network, which was eventually used to detect solid soil and pore space in CT images. Chauhan et al. (2016b) extended that approach to 3D greyscale values. Moreover, they compared the performances of seven machine learning algorithms (either unsupervised and supervised) to segment four $\mu$-CT imaged samples, concluding that the use of K-means to guide the construction of the feature vector dataset for least-square support vector machine led to the best results (Chauhan et al. 2016a). Berg et al. (2018) employed Trainable WEKA Segmentation (TWS) (Arganda-Carreras et al. 2017) and no filtering to segment an artificially generated 2D orthogonal image of a Bentheimer sandstone sample. This processing pipeline was found to be more accurate and more robust to image noise and artefacts when compared with more traditional pipelines involving the sequential use of a filtering and a segmentation algorithm. Results shown in Andrew (2018) are in agreement with those of Berg et al. (2018).

In this study, the Trainable WEKA Segmentation 3D (TWS) plugin of the open-source image processing software ImageJ, available in its Fiji distribution (Schindelin et al. 2012), was employed. The underlying concept to the method consists in the generation of multiple modalities of the original image by applying nonlinear filters. This step is followed by a classification problem based on user generated training data, which are employed to train one of the available classifier algorithms. A fast random forest classifier (Supek 2008) was chosen among the pool of options available; this a recent more efficient variation of random decision forest algorithms (Ho 1994; Breiman 2001). The training of the classifier 
is performed making use of the data manually labelled by the user, on the basis of the features selected. The first step of the process is the selection of the phases to segment. The user then manually labels data, assigning selected regions of the images to each of the phases chosen. The features of the input image are then computed on the different modalities of the image generated by applying the selected nonlinear filters and feature vectors are extracted to train the classifier. In this work, the features selected for the training and the classification tasks were the mean and variance of each voxel. Both of these features are defined over a cubic region defined in the neighbourhood of each voxel, within a distance, ranging from 1 to 8 voxels from the position of the central voxel considered. For each feature, either mean or variance, and for each length value, a new image is created, where each voxel value corresponded to the mean (or variance) calculated over the region of the image defined by the length considered. Since machine learning based segmentation has proven to be robust to image noise (Berg et al. 2018; Andrew 2018), no filtering was used prior to segmentation.

\subsection{Estimation of Rock Properties}

Porosity, specific surface area and single-phase flow permeability were computed and compared for all the images considered. Porosity was computed as the ratio between the pore space and the bulk volume of the image. Specific surface area was defined as the interfacial area between the pore space and the rock matrix, divided by rock matrix volume. All the volume and area measurements were performed by employing the label analysis toolbox available in Thermofisher Avizo 9.5. Single-phase flow permeability was computed running direct numerical simulations through the connected pore space. A pressure drop of $1 \mathrm{~Pa}$ was imposed, considering two opposite faces of the cubic sample as inlet and outlet boundaries, respectively. A solver available in openFOAM was employed (Raeini et al. 2012).

To compare results computed from the images segmented using the two segmentation pipelines, we used the percentage difference between two estimates of the same property in a dataset,

$$
d_{x}=\frac{x_{\mathrm{W}}-x_{\mathrm{TWS}}}{x_{\mathrm{W}}} \times 100
$$

where $x_{\mathrm{W}}$ is the estimate of the pore space property $x$ obtained by employing the watershed segmentation, and $x_{\text {TWS }}$ is the estimate made by employing Trainable WEKA Segmentation 3D.

\subsection{In Situ Contact Angle and Fluid-Fluid Interfacial Curvature Measurements}

The greyscale image of the trapped decane ganglion in a Ketton limestone pore was segmented with the processing pipelines 1 and 2 . In order to perform contact angle measurements, the three-phase contact line between decane, brine and rock walls was identified by employing the Thermofisher Avizo Fire 9.5 label interface toolbox. We then employed the algorithm proposed by Scanziani et al. (2017) to automatically measure the contact angles formed by the two fluids sitting on the rock matrix, along that contact line. The parameters chosen in the algorithm were the same for both the segmented images analysed. 
To compute fluid-fluid interfacial mean curvature, the interfacial area between the two fluids was identified. In order to exclude from our analysis values of curvature measurements performed in regions of the fluid-fluid interface close to rock walls, we followed the approach suggested in Singh et al. (2016). A dilation of three voxels was performed on the rock matrix label. The dilated rock label image was used to mask the three-phase segmented image, to obtain a third image where only fluid labels were present. This final image was used to reconstruct the fluid-fluid interface surface by means of a marching cube algorithm implemented in Thermofisher Avizo Fire 9.5. The reconstructed surface was smoothed (smoothing extent parameter equal to 3 ) to correct for the effect of voxelization of the actual interface shape. Local mean curvature measurements were eventually performed.

\subsection{Mineral Topological Characterization}

In order to choose the minerals to be segmented in the subsample of the Berea sandstone image obtained by X-ray $\mu$-CT, scanning electron microscopy (SEM) was employed to aid mineral identification. A second specimen from the same core of the one imaged was used to create the SEM imagery. SEM operated in back-scattered electron (BEC) mode coupled with energy-dispersive X-ray spectroscopy (EDS) allowed for the identification of the main mineral groups present.

The $500^{3}$ voxels greyscale image was thus processed with the two pipelines assessed in this work. SEM + EDS was employed as a qualitative tool to aid the selection of the mineral phases to segment in the X-ray $\mu$-CT image. Through SEM + EDS the main mineral groups identified were: clay minerals, quartz, feldspar, calcite cementation and non-specified minerals embedding heavy metal compounds. On the basis of SEM + EDS results and previous work by Lai et al. (2015), we chose to segment the CT image into six phases: pore space, clay minerals, quartz, feldspar group minerals, calcite cementation and others highly attenuating minerals. On the other hand, a closer look to the CT greyscale image, revealed that only five of the six phases initially chosen showed clear differences in their average greyscale value. In particular, the greyscale values belonging to the grains reconcilable to quartz and feldspar minerals were very similar. Therefore, it was chosen to reduce the number of the phases to segment to five: pore space, clay minerals, quartz-feldspar minerals, calcite cementation and other highly attenuating minerals. Finally, two segmented images were obtained, one for each segmentation algorithm employed.

From the two segmented images, volume as well as mineral-to-pore surface areas was computed. The volume fractions were computed as the ratio between the total volume of each phase to the total bulk volume. Mineral-to-pore surface areas measured the total surface area that a certain mineral group shared with the pore space. The interfaces between each mineral phase and the pore space were thus identified and their area was computed by employing Thermofisher Avizo Fire 9.5 software. Finally, mineral-to-pore surface area fractions were computed as the ratio between each mineral-to-pore surface area and the total surface area shared by the pore space and the entire rock matrix. All the area and volume measurements were performed with the label analysis package available in Thermofisher Avizo Fire 9.5. 


\section{Results and Discussion}

\subsection{Sensitivity of Porosity, Specific Surface Area and Single-Phase Flow Permeability}

Figure 1 shows the greyscale cross sections of the datasets considered for this analysis. For each rock, the figure also shows the differential images obtained by subtracting to each other the segmented images obtained with the two processing pipelines. Misclassified voxels are mainly concentrated in the boundary regions between the pore space and the rock matrix.

Figure 2 reports the estimates of porosity, specific surface area and single-phase flow permeability, while Table 2 shows the relative differences for each property and each sample. In all samples but Berea sandstone, porosity estimates show smaller differences compared to other properties. The smallest difference in porosity is observed for Bentheimer sandstone, while the largest is observed for the Berea sandstone. Trainable WEKA Segmentation 3D systematically overestimates the fraction of rock matrix compared to the watershed segmentation in all samples but Estaillades limestone.

Bentheimer sandstone shows the least variation in the estimates for specific surface area and single-phase flow permeability. In contrast, the largest discrepancy in specific surface area is observed for Edwards limestone, while Berea sandstone shows the largest difference for estimates of single-phase flow permeability. This is consistent with the observed sensitivity for porosity. Our observations that Berea sandstone porosity and single-phase flow permeability estimates are very sensitive to image processing are consistent with observations in Leu et al. (2014).

The relative differences are larger for single-phase flow permeability than porosity and specific surface area. In attempt to identify the sources of such large sensitivities for estimates of permeability, a maximum ball extraction (Raeini et al. 2017) was performed on each segmented image, and pore and throat radii distributions are obtained. Although the shape of the distributions are similar, small shifts towards larger values of pore and throat radii distributions are observed when permeability increased. Single-phase flow permeability estimates are found sensitive to these changes. These changes may thus strongly affect the overall connectivity of the pore space. For reference, Fig. 3 reports the pore and throat radii distributions for the two lithologies exhibiting the largest variation in single-phase flow permeability estimates, i.e. Berea sandstone and Edwards limestone.

The observation that single-phase flow permeability relative differences are larger than those observed for porosity and surface area suggests that the sensitivity of an estimate to the image processing pipeline increases with the complexity of the interpretation. An analogous conclusion is suggested by the results obtained from the computation of contact angle and fluid-fluid interfacial curvature measurements.

\subsection{Sensitivity of In Situ Contact Angle and Fluid-Fluid Interfacial Curvature Measurements}

The three-phase contact lines identified in the two segmented images and their difference are shown in Fig. 4. There are significant differences between the segmentation pipelines, and contact angle measurements are unsurprisingly dissimilar. The mean and standard deviation of the measurements obtained in the two cases are reported in Table 3, while the 


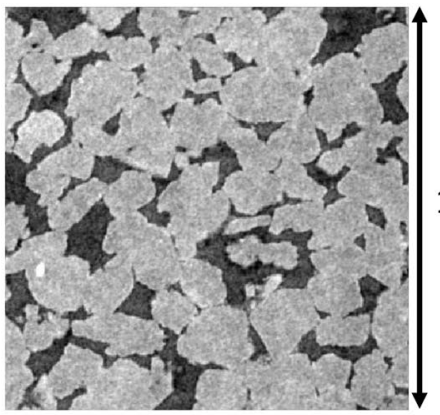

(a) Bentheimer sandstone

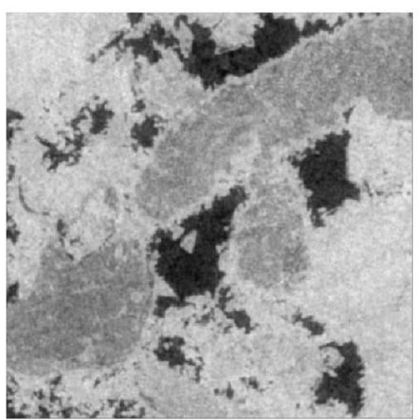

(b) Estaillades limestone

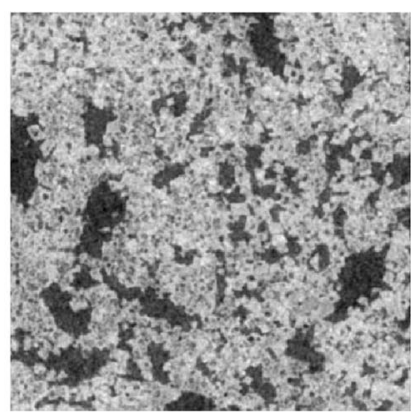

(c) Edwards limestone

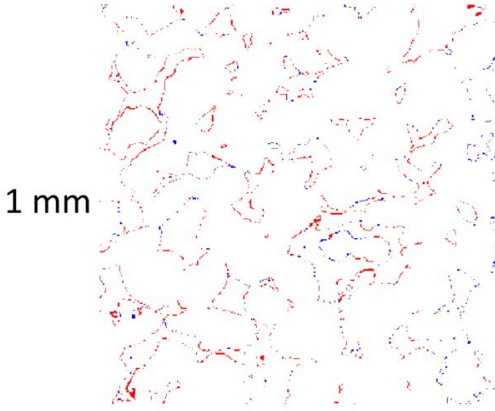

(d) Difference in the segmented images

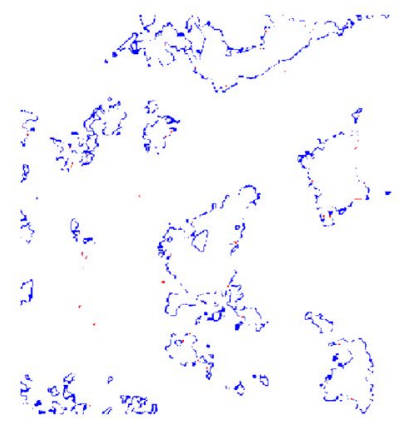

(e) Difference in the segmented images

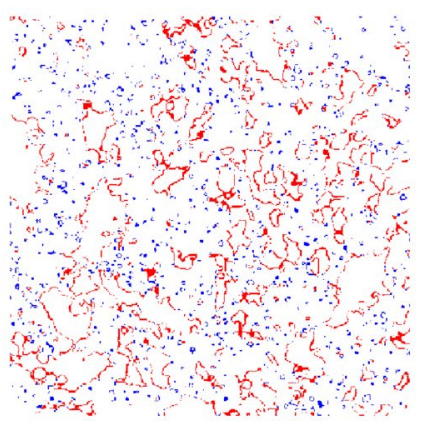

(f) Difference in the segmented images

Watershed rock phase larger than TWS rock phase

TWS rock phase larger than Watershed rock phase

Fig. 1 Cross sections of three of the five samples considered for the analysis of pore space properties sensitivity to image processing. The voxel size is $2.5 \mu \mathrm{m}$. The rocks are, respectively: a Bentheimer sandstone; b Estaillades limestone; c Edwards limestone and the differential images obtained by subtracting the respective segmented images with the two processing pipelines considered

relative frequency distributions for the contact angle measurements are reported in Fig. 5. The difference in the mean and the standard deviation are $5.43^{\circ}$ and $3.46^{\circ}$, respectively. Moreover, the large difference in the estimated skewness parameter for the two distributions (Table 3) confirms that the two distributions are distinct. The mean and standard 
Fig. 2 Pore space properties estimates for the five rock samples considered: a Porosity $(\phi[-])$; b Specific surface area ( $\left.A_{s}[1 / \mathrm{mm}]\right)$; c Single-phase flow permeability $(K[\mathrm{mD}]$, $\left.1 \mathrm{mD}=9.869233 \times 10^{-16} \mathrm{~m}^{2}\right)$. Estimates are shown for both the segmentation approaches under investigation. The largest difference in estimates is observed for single-phase flow permeability

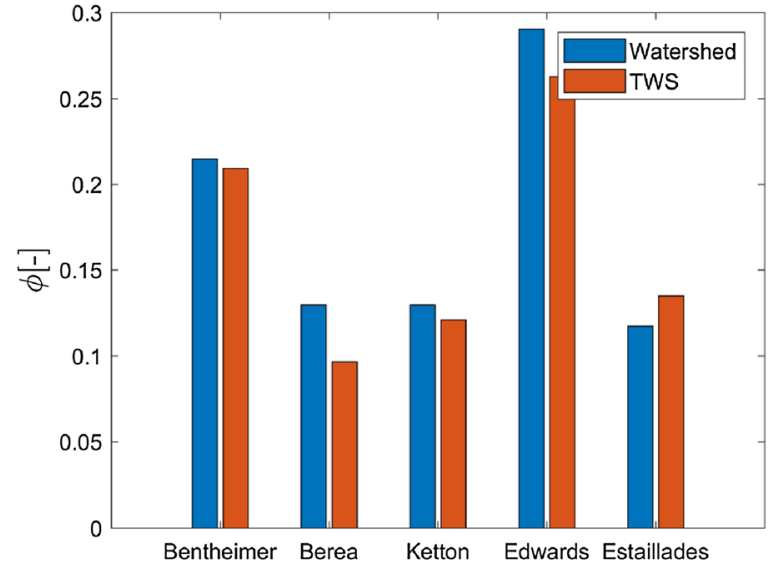

(a)

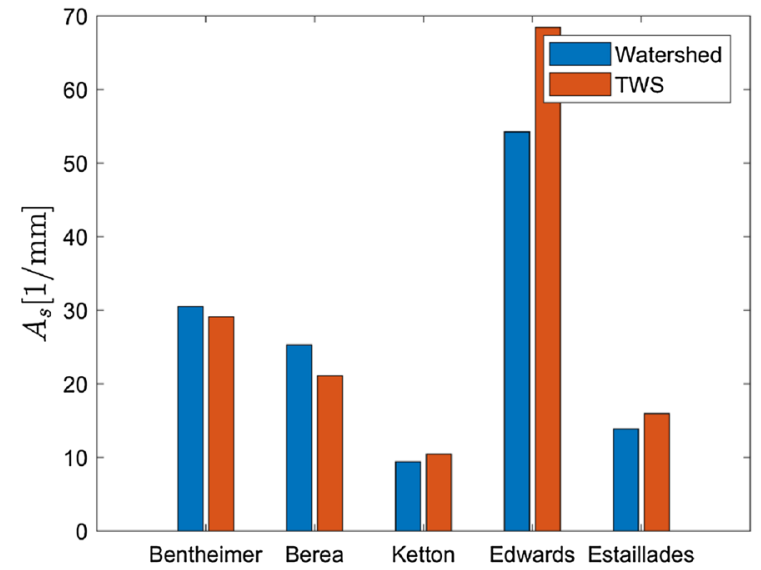

(b)

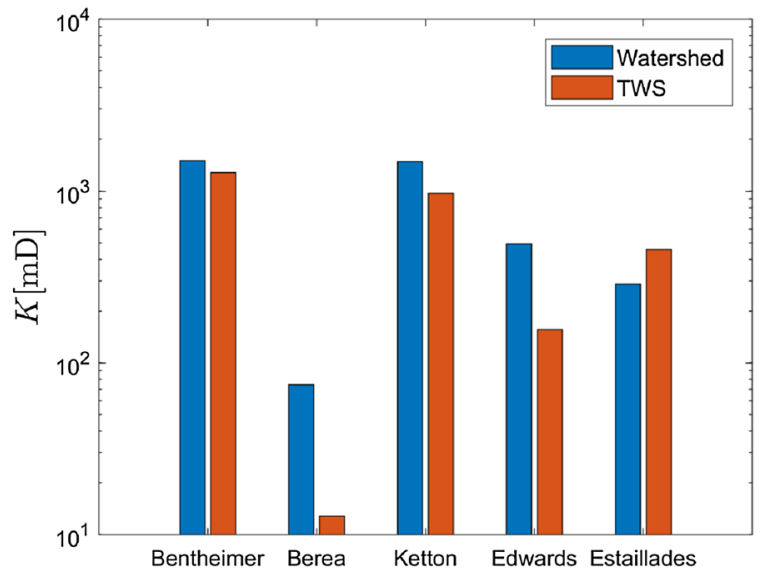

(c) 
Table 2 Percentage difference $\left(d_{x}\right)$ of the estimates of porosity $(\phi)$ specific surface area $\left(A_{s}\right)$ and single-phase flow permeability $(K)$ obtained from images segmented with the two processing pipelines considered

\begin{tabular}{lrrr}
\hline & \multicolumn{1}{c}{$d_{\phi}[\%]$} & \multicolumn{1}{c}{$d_{A_{s}}[\%]$} & \multicolumn{1}{c}{$d_{K}[\%]$} \\
\hline Bentheimer & 2.638 & 4.666 & 14.473 \\
Berea & 25.555 & 16.497 & 82.891 \\
Ketton & 6.516 & -10.799 & 34.348 \\
Edwards & 9.500 & -26.158 & 68.422 \\
Estaillades & -14.847 & -14.847 & -58.876 \\
\hline
\end{tabular}

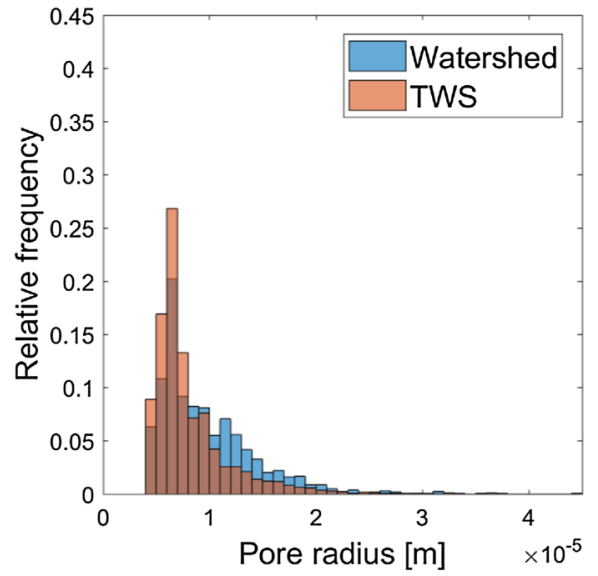

(a) Berea sandstone

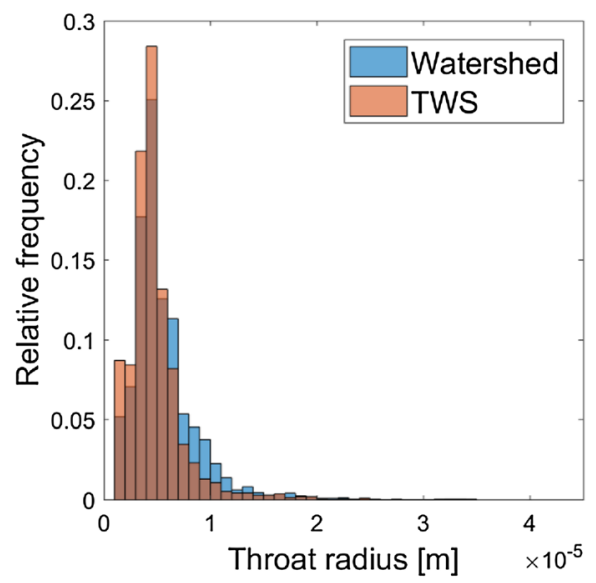

(c) Berea sandstone

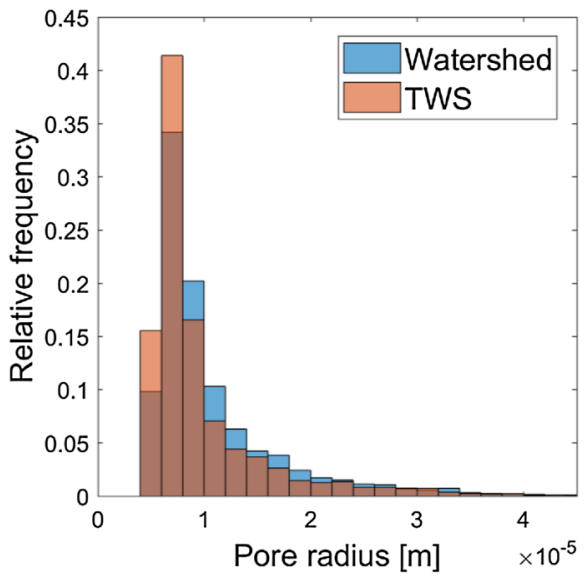

(b) Estaillades limestone

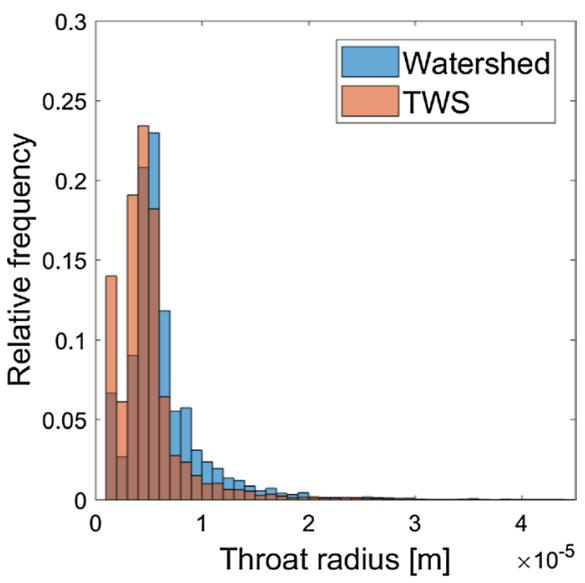

(d) Estaillades limestone

Fig. 3 Comparison of the pore and throat radii distributions for Berea sandstone and Edwards limestone. For these samples, Trainable WEKA Segmentation 3D (TWS) segmentation leads to pore and throat radii distributions shifted towards smaller values. These changes explain the larger single-phase flow permeability estimates obtained from watershed segmented images 


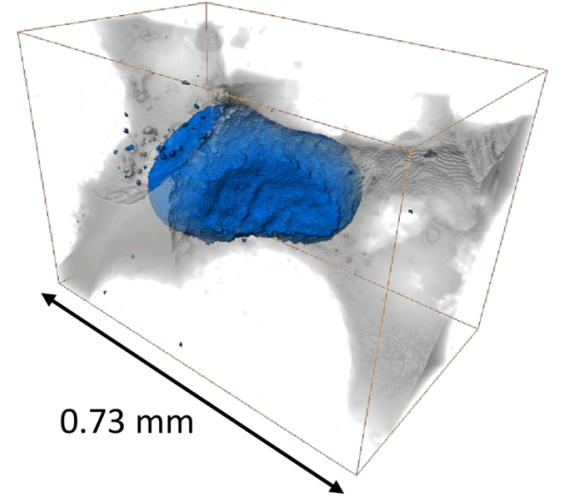

(a) Trapped decane ganglion

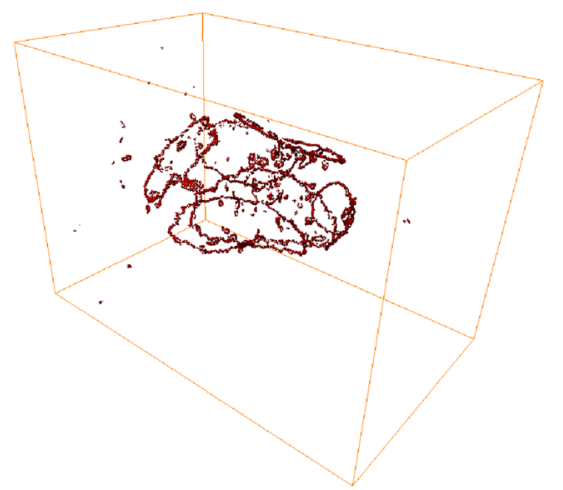

(c) TWS three-phase contact line

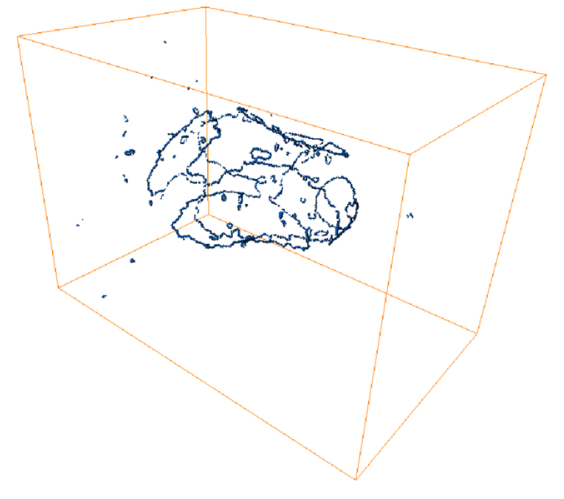

(b) Watershed three-phase contact line

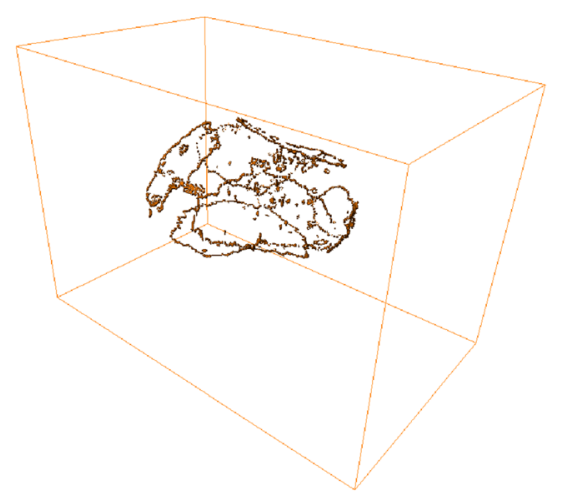

(d) Mismatch in three-phase contact line identification

Fig. 4 Three-phase contact lines were identified in segmented images to measure contact angles: a Volume rendering of the segmented trapped decane ganglion; b Contact lines identified in the image processed by non-local means filtering and watershed segmentation; c Contact line in the image processed by Trainable WEKA Segmentation 3D (TWS); d Differential image of the two contact lines

deviation of the contact angle distribution obtained for the TWS segmented image appear to be influenced by a few very large contact angle observations. The first possible reason for these observations could be that the three-phase contact line identified in the TWS segmented image is less smooth than the one identified in the watershed segmented image. The second possibility is the presence of artefacts caused by misidentification of the correct angle to measure, i.e. the algorithm measured the contact angle on the side of the nonwetting phase rather than the wetting one. The third, pinning (if present) of the oil-brine interface in the roughness of the rock, which was smoothed more by one segmentation algorithm than the other.

The measurements we obtained in the watershed segmented image differ from those (Scanziani et al. 2017) obtained on the very same dataset, because of the different similarity value chosen for the non-local means filtering and the subjective choice of phase thresholds in applying watershed segmentation. Measurements obtained in the TWS segmented image further overestimate the mean contact angle. The mean values obtained in this study 


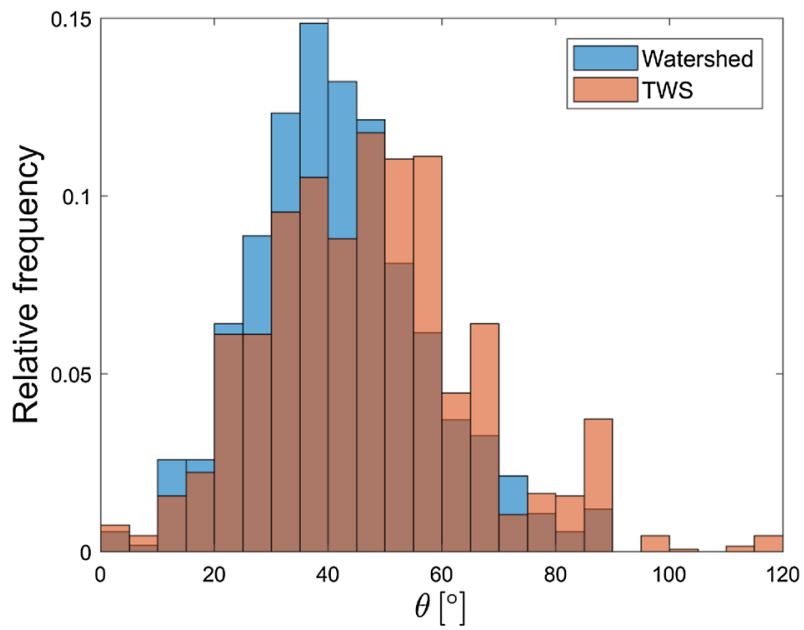

(a)

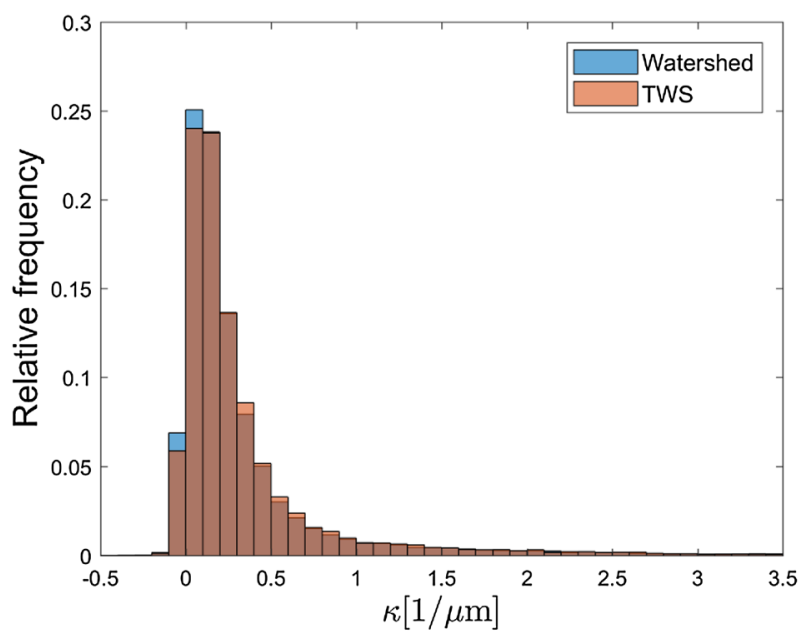

(b)

Fig. 5 Relative frequency distributions associated with the measurements of contact angle $\theta\left[{ }^{\circ}\right]$ and fluidfluid interfacial mean curvature $\kappa[1 / \mu \mathrm{m}]$ from the same greyscale image segmented with watershed segmentation and Trainable WEKA Segmentation 3D (TWS)

Table 3 Results of contact angle $(\theta)$ and fluid-fluid interfacial curvature $(\kappa)$ measurement, computed on the same image segmented through two different image processing pipelines

\begin{tabular}{lcrr}
\hline & Watershed & TWS & \multicolumn{1}{c}{$d_{x}[\%]$} \\
\hline Mean $\theta\left[^{\circ}\right]$ & 41.873 & 47.301 & -12.964 \\
St.Dev $\theta\left[^{\circ}\right]$ & 15.299 & 18.762 & -22.638 \\
Skewness $\theta\left[^{\circ}\right]$ & 0.397 & 0.517 & -30.176 \\
Mean $\kappa[1 / \mu \mathrm{m}]$ & 0.455 & 0.494 & -8.617 \\
SD $\kappa[1 / \mu \mathrm{m}]$ & 1.178 & 1.335 & -13.301 \\
Skewness $\kappa[1 / \mu \mathrm{m}]$ & 7.595 & 7.913 & -4.196
\end{tabular}


as well as those observed in Scanziani et al. (2017) are consistent with those of a water-wet rock.

On the other hand, the differences observed for the measurements of fluid-fluid interfacial curvature are much smaller. The difference in the mean value is $0.3 \mu \mathrm{m}^{-1}$, while the difference in standard deviation is $0.15 \mu \mathrm{m}^{-1}$. The relative frequency distributions associated with local mean curvature measurements (reported in Fig. 5) show agreement, as confirmed by the very small difference in their skewness parameter (Table 3 ). Their shapes are similar, with a similar tailing of the distributions with increasing mean curvature.

The outcome of this analysis suggests that interfacial curvature measurement is less sensitive to the image processing workflow than the meausurement of contact angle. As expected, this is a result of fluid-fluid interfaces being smooth surfaces defined over a larger number of voxels as opposed to the three-phase contact lines. On the other hand, contact angles are measured close to the point where three different phases meet, so that partial volume effects and resolution limits are more important (Leu et al. 2014; Soulaine et al. 2016; Saxena et al. 2017). Moreover, additional complications in performing the measurement are added by the rock rough edges, which are the regions of an image exhibiting the largest variability when segmented, due to various levels of resolvable and sub-resolution roughness (AlRatrout et al. 2018). By comparing the results obtained for contact angle measurements and fluid-fluid interfacial curvature, we observe again that the sensitivity to the choice of the image processing pipeline increases with the complexity in the definition and interpretation of the quantity computed from the images.

\subsection{Sensitivity of the Rock Mineralogy Descriptors}

The Berea sandstone tomogram was segmented into five phases: pore space, clay minerals, quartz-feldspar minerals, calcite cementation, others highly attenuating minerals (Fig. 6). Figure 6 also shows that the segmented clay phase can include regions of the rock matrix that present sub-resolution porosity, which assume a greyscale intensity similar to that of clay minerals due to partial volume effect. The volume renderings of each phase segmented are shown in Fig. 7. They visually demonstrate the complexity of the topology of chemical heterogeneities in natural porous media.

Table 4 reports the results obtained for the computation of the volume fraction of each phase. The sensitivity of these quantities are small compared to what was observed for other quantities previously analysed in this study. Analogous results are observed for mineral-to-pore surface area fractions (Table 5). On the other hand, a substantial difference is observed in the mineral-to-pore surface area estimates (Table 6), with much larger interfaces between clay, quartz-feldspar, calcite cementation and the pore space in the image segmented by Trainable WEKA Segmentation 3D. This suggests that the interfaces identified by Trainable WEKA Segmentation 3D are much rougher than those identified by watershed algorithm. Such a large variation of the total exposed surface area may have significant implications in the context of reactive transport modelling.

This finding suggests once more that the sensitivity to image processing is larger when the property of interest is more complex to derive. The sensitivity to image processing seems to become more and more relevant when the definition of the property of interest is more closely related to phase boundary shape. Indeed, the same has already been observed for single-phase flow permeability, where boundary conditions and geometry contribute to the description of the interaction between solid walls and the fluid; the same has also been 


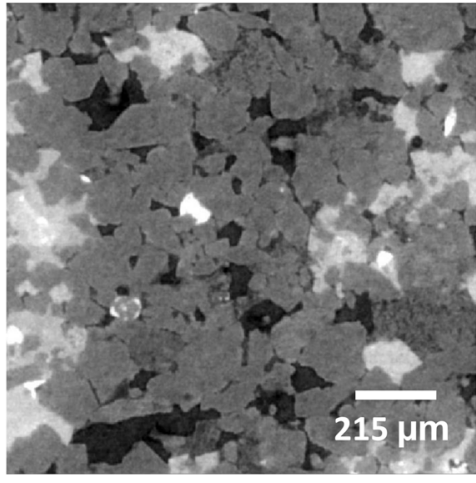

(a) Berea greyscale image

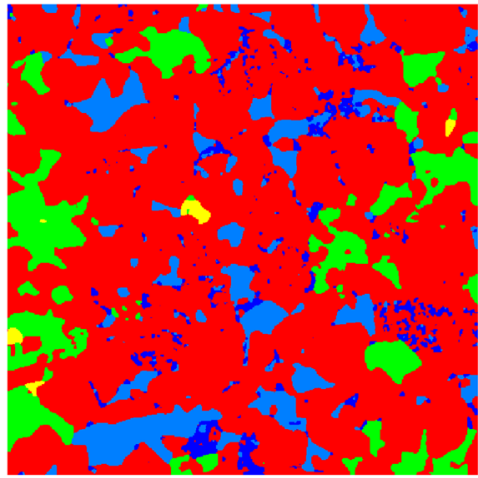

(c) Berea watershed segmentation

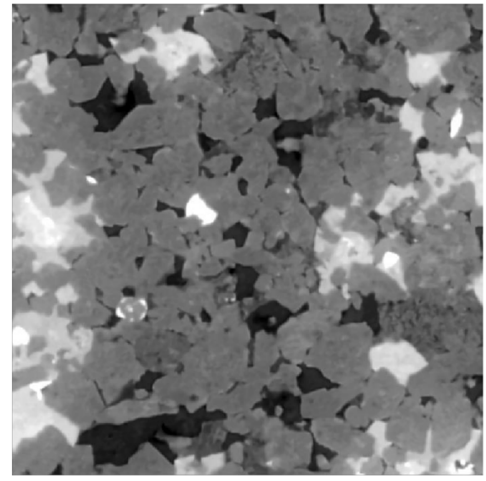

(b) Berea filtered image

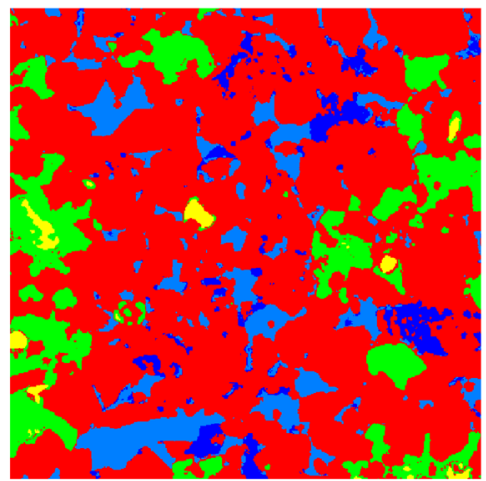

(d) Berea TWS segmentation

Fig. 6 Orthogonal slices of the $500^{3}$ voxels CT image of a Berea sandstone. Watershed segmentation was performed on the non-local means filtered image, while Trainable WEKA Segmentation 3D (TWS) was employed on the greyscale unfiltered one

observed for contact angle measurement, which definition relies of the identification of the contact line between three different phases.

\subsection{Wider Implications}

A broad assessment of the results suggests that the sensitivity of the properties of interest increases with the complexity of the interpretation of the observation and also with the complexity of the rock structure. Porosity and mineral volume fractions were the properties affected the least by the choice of the processing pipeline. We hypothesize that this is a consequence of their definitions: both definitions rely on calculations performed over larger regions 


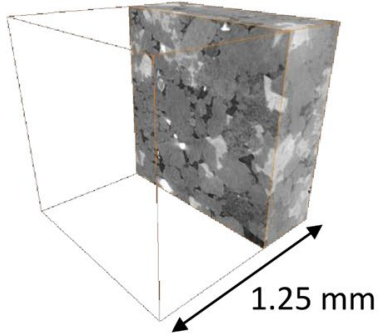

(a) Berea sandstone: greyscale

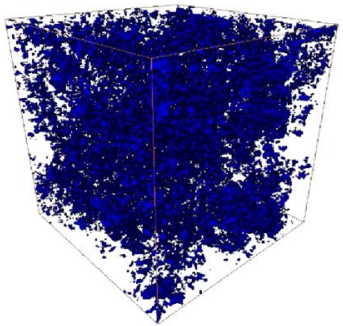

(c) Clay volume

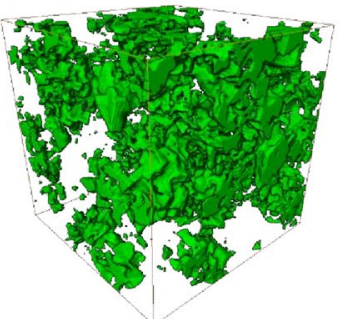

(e) Calcite volume

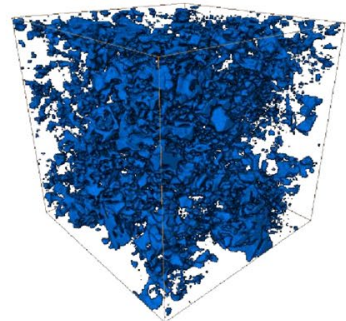

(b) Pore space volume

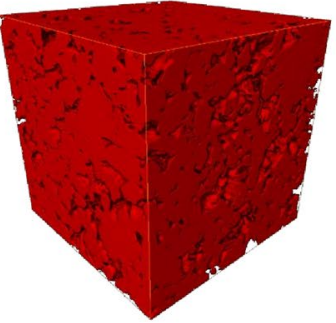

(d) Quartz-Feldspar volume

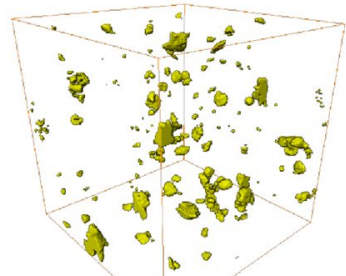

(f) Other minerals volume

Fig. 7 Volume renderings of the $500^{3}$ voxels CT image of a Berea sandstone: greyscale images and segmented phases upon employment of non-local means filtering and watershed segmentation

Table 4 Volume fraction of each phase in the Berea sandstone images segmented employing the two image processing pipelines under investigation. The volume fraction was defined as the ratio between the volume of each phase to the total volume in the image

\begin{tabular}{lllr}
\hline & Watershed $[-]$ & TWS $[-]$ & $d_{x}[\%]$ \\
\hline Pore & 0.079 & 0.081 & -3.136 \\
Clay & 0.038 & 0.039 & -4.546 \\
Quartz-Feldspar & 0.771 & 0.746 & 3.215 \\
Calcite & 0.109 & 0.123 & -13.397 \\
Others & 0.004 & 0.010 & -140.538
\end{tabular}


Table 5 Surface area fraction of the interface between each mineral and the pore space (mineral-to-pore surface area fraction) calculated in the segmented Berea sandstone images obtained upon employment of the two image processing pipelines. Surface area fraction has been defined as the ratio between the surface area of the interface shared by each mineral and the pore space and the total surface area of the interface shared by the pore space with the whole rock matrix

\begin{tabular}{lllr}
\hline & Watershed [-] & TWS $[-]$ & \multicolumn{1}{c}{$d_{x}[\%]$} \\
\hline Clay & 0.311 & 0.308 & 0.839 \\
Quartz-Feldspar & 0.656 & 0.642 & 2.092 \\
Calcite & 0.031 & 0.048 & -58.299 \\
Others & 0.003 & 0.002 & 46.303 \\
\hline
\end{tabular}

Table 6 Surface area of the interface between each mineral and the pore space-mineral-to-pore surface area-calculated in the segmented Berea sandstone images obtained with watershed and Trainable WEKA Segmentation 3D (TWS)

\begin{tabular}{lccr}
\hline & Watershed $\left[\mathrm{mm}^{2}\right]$ & TWS $\left[\mathrm{mm}^{2}\right]$ & $d_{x}[\%]$ \\
\hline Clay & 16.669 & 22.041 & -32.224 \\
Quartz-Feldspar & 35.195 & 45.948 & -30.552 \\
Calcite & 1.643 & 3.468 & -111.080 \\
Others & 0.176 & 0.126 & 28.399 \\
\hline
\end{tabular}

of the images. This reduces the relative importance of phase boundary identification, a sensitive task of image processing. For similar reasons, if one considers a porous medium filled with two fluids, exhibiting a complete wetting/non-wetting situation, we can expect the nonwetting phase to be less sensitive to image processing because it is mainly located in the centre of big pores. The wetting phase, however, is then more affected because resides in pore corners, small pores and sub-resolution pores.

In situ contact angle estimation relies on the precise identification of both fluid-fluid and fluid-solid boundaries, both situated in the most challenging regions of the images. Moreover, partial volume effects are significantly amplified by the requirement of a three-phase boundary. As a consequence, the combination of two potential sources of errors may lead to appreciably inconsistent measurements. In the same way, single-phase flow permeability estimation suffers from issues related to boundary identification. This is particularly relevant for rocks in which smaller throats constitute an important contribution to connectivity. Moreover, although we did not investigate the role of voxel size on image segmentation results, it is well known that it controls the estimation of petrophysical properties (Combaret et al. 2013a, b). Consequently, we expect quantities whose computation is strongly related to smaller features in the images, such as contact angle measurement and single-phase flow permeability, to be sensitive to voxel size as well.

\section{Conclusions}

We presented an assessment and a comparison of the quantitative results obtained from $\mathrm{X}$-ray $\mu$-CT images segmented using two different image processing approaches: non-local means filtering followed by watershed segmentation; machine learning based segmentation 
with a fast random forest classifier (Ho 1994; Breiman 2001). In order to assess the relative importance of the image processing pipeline selected, diverse datasets and properties to compute were considered. In particular, the focus of this work was to extend our understanding of sensitivity of image processing to the estimation of multiphase fluid properties (in situ contact angle, interfacial curvature) and mineral phase segmentation.

Porosity, specific surface area and single-phase flow permeability were estimated in ten two-phase (rock matrix and pore space) segmented images. Single-phase flow permeability proved to be the most sensitive property to the processing pipeline chosen.

Contact angles and fluid-fluid interfacial curvature were measured in an image of a trapped decane ganglion in a water-wet Ketton limestone pore (Singh and Blunt 2018). Measurements of contact angle, while comparable to past studies, were sensitive to image processing workflows. On the contrary, measurements of fluid-fluid interfacial curvature were less sensitive to image processing workflows.

Four mineral groups and the pore space were segmented in a Berea sandstone. Estimates of volume fractions as well as mineral-to-pore surface area fractions were insensitive to image processing workflows. The same was not true for mineral-to-pore surface area, which suggests that the choice of the processing pipeline may be particularly relevant for reactive transport modelling applications.

The greatest sensitivities in image processing arise in the identification of boundaries where partial volume and resolution effects may be significant. Sensitivity increases with increasing dependence on boundary identification and decreasing availability of the necessary boundaries in the image.

Acknowledgements Funding was provided by the Shell Digital Rocks Programme at Imperial College London.

Open Access This article is licensed under a Creative Commons Attribution 4.0 International License, which permits use, sharing, adaptation, distribution and reproduction in any medium or format, as long as you give appropriate credit to the original author(s) and the source, provide a link to the Creative Commons licence, and indicate if changes were made. The images or other third party material in this article are included in the article's Creative Commons licence, unless indicated otherwise in a credit line to the material. If material is not included in the article's Creative Commons licence and your intended use is not permitted by statutory regulation or exceeds the permitted use, you will need to obtain permission directly from the copyright holder. To view a copy of this licence, visit http://creativecommons.org/licenses/by/4.0/.

\section{References}

Alhammadi, A.M., AlRatrout, A., Singh, K., Bijeljic, B., Blunt, M.J.: In situ characterization of mixedwettability in a reservoir rock at subsurface conditions. Sci. Rep. 7(1), 10753 (2017). https://doi. org/10.1038/s41598-017-10992-w

Alhosani, A., Scanziani, A., Lin, Q., Pan, Z., Bijeljic, B., Blunt, M.J.: In situ pore-scale analysis of oil recovery during three-phase near-miscible $\mathrm{CO}_{2}$ injection in a water-wet carbonate rock. Adv. Water Resour. 134, 103432 (2019). https://doi.org/10.1016/j.advwatres.2019.103432

AlRatrout, A., Raeini, A.Q., Bijeljic, B., Blunt, M.J.: Automatic measurement of contact angle in pore-space images. Adv. Water Resour. 109, 158-169 (2017). https://doi.org/10.1016/j.advwatres.2017.07.018

AlRatrout, A., Blunt, M.J., Bijeljic, B.: Wettability in complex porous materials, the mixed-wet state, and its relationship to surface roughness. Proc. Natl. Acad. Sci. 115(36), 8901-8906 (2018). https://doi. org/10.1073/pnas.1803734115

Andersson, M., Beale, S.B., Espinoza, M., Wu, Z., Lehnert, W.: A review of cell-scale multiphase flow modeling, including water management, in polymer electrolyte fuel cells. Appl. Energy 180, 757-778 (2016). https://doi.org/10.1016/j.apenergy.2016.08.010 
Andrew, M.: A quantified study of segmentation techniques on synthetic geological XRM and FIB-SEM images. Comput. Geosci. 22, 1503 (2018). https://doi.org/10.1007/s10596-018-9768-y

Andrew, M., Bijeljic, B., Blunt, M.J.: Pore-scale imaging of geological carbon dioxide storage under in situ conditions. Geophys. Res. Lett. 40(15), 3915-3918 (2013). https://doi.org/10.1002/grl.50771

Andrew, M., Bijeljic, B., Blunt, M.J.: Pore-scale contact angle measurements at reservoir conditions using X-ray microtomography. Adv. Water Resour. 68, 24-31 (2014). https://doi.org/10.1016/j.advwatres.2014.02.014

Andrew, M., Bijeljic, B., Blunt, M.J.: Pore-scale imaging of trapped supercritical carbon dioxide in sandstones and carbonates. Int. J. Greenhouse Gas Control 22, 1-14 (2014b)

Arganda-Carreras, I., Kaynig, V., Rueden, C., Eliceiri, K.W., Schindelin, J., Cardona, A., Seung, H.S.: Trainable Weka Segmentation: a machine learning tool for microscopy pixel classification. Bioinformatics 33(15), 2424-2426 (2017). https://doi.org/10.1093/bioinformatics/btx180

Armstrong, R.T., Pentland, C.H., Berg, S., Hummel, J., Lichau, D., Bernard, L., Group, V.S.: Estimation of curvature from micro-CT liquid-liquid displacement studies with pore scale resolution, pp 1-6 (2012)

Berg, S., Saxena, N., Shaik, M., Pradhan, C.: Generation of ground truth images to validate micro-CT imageprocessing pipelines. Lead. Edge 37(6), 412-420 (2018). https://doi.org/10.1190/tle37060412.1

Beucher, S., Meyer, F.: The morphological approach to segmentation: the watershed transformation. Math. Morphol. Image Process. 34, 433-481 (1993). https://doi.org/10.1201/9781482277234-12

Blunt, M.J.: Multiphase Flow in Permeable Media. Cambridge University Press, Cambridge (2017). https://doi. org/10.1017/9781316145098

Blunt, M.J., Bijeljic, B., Dong, H., Gharbi, O., Iglauer, S., Mostaghimi, P., Paluszny, A., Pentland, C.: Porescale imaging and modelling. Adv. Water Resour. 51, 197-216 (2013). https://doi.org/10.1016/j.advwa tres.2012.03.003

Breiman, L.: Machine Learning. Kluwer Academic Publishers, Dordrecht (2001). https://doi. org/10.1023/A:101093340

Buades, A., Coll, B., Morel, J.: A non-local algorithm for image denoising. In: 2005 IEEE Computer Society Conference on Computer Vision and Pattern Recognition (CVPR'05), vol. 2, pp. 60-65 (2005). https:// doi.org/10.1109/CVPR.2005.38

Bultreys, T., Boone, M.A., Boone, M.N., De Schryver, T., Masschaele, B., Van Hoorebeke, L., Cnudde, V.: Fast laboratory-based micro-computed tomography for pore-scale research: Illustrative experiments and perspectives on the future. Adv. Water Resour. 95, 341-351 (2016a). https://doi.org/10.1016/j.advwa tres.2015.05.012

Bultreys, T., De Boever, W., Cnudde, V.: Imaging and image-based fluid transport modeling at the pore scale in geological materials: a practical introduction to the current state-of-the-art. Earth Sci. Rev. 155, 93-128 (2016). https://doi.org/10.1016/j.earscirev.2016.02.001

Chauhan, S., Rühaak, W., Anbergen, H., Kabdenov, A., Freise, M., Wille, T., Sass, I.: Phase segmentation of X-ray computer tomography rock images using machine learning techniques: an accuracy and performance study. Solid Earth 7(4), 1125-1139 (2016). https://doi.org/10.5194/se-7-1125-2016

Chauhan, S., Rühaak, W., Khan, F., Enzmann, F., Mielke, P., Kersten, M., Sass, I.: Processing of rock core microtomography images: using seven different machine learning algorithms. Comput. Geosci. 86, 120128 (2016b). https://doi.org/10.1016/j.cageo.2015.10.013

Combaret, N., Dvorkin, J., Glatt, E., Han, J., Kabel, M., Keehm, Y., Krzikalla, F., Lee, M., Madonna, C., Marsh, M., Mukerji, T., Saenger, E.H., Sain, R., Saxena, N., Ricker, S., Wiegmann, A., Zhan, X.: Digital rock physics benchmarks-part I: imaging and segmentation. Comput. Geosci. 50, 25-32 (2013a). https://doi. org/10.1016/j.cageo.2012.09.005

Combaret, N., Dvorkin, J., Glatt, E., Han, J., Kabel, M., Keehm, Y., Krzikalla, F., Lee, M., Madonna, C., Marsh, M., Mukerji, T., Saenger, E.H., Sain, R., Saxena, N., Ricker, S., Wiegmann, A., Zhan, X.: Digital rock physics benchmarks-part II: computing effective properties. Comput. Geosci. 50, 33-43 (2013b). https:// doi.org/10.1016/j.cageo.2012.09.008

Cortina-Januchs, M.G., Quintanilla-Dominguez, J., Vega-Corona, A., Tarquis, A.M., Andina, D.: Detection of pore space in CT soil images using artificial neural networks. Biogeosciences 8(2), 279-288 (2011). https ://doi.org/10.5194/bg-8-279-2011

Debe, M.K.: Electrocatalyst approaches and challenges for automotive fuel cells. Nature 486(7401), 43-51 (2012). https://doi.org/10.1038/nature11115

Ho, T.K.: Random decision forests. In: Proceedings of 3rd International Conference on Document Analysis and Recognition, vol. 1, pp. 278-282 (1994). https://doi.org/10.1109/ICDAR.1995.598994

Iassonov, P., Gebrenegus, T., Tuller, M.: Segmentation of X-ray computed tomography images of porous materials: a crucial step for characterization and quantitative analysis of pore structures. Water Resour. Res. 45, 1-12 (2009). https://doi.org/10.1029/2009WR008087

Kaestner, A., Lehmann, E., Stampanoni, M.: Imaging and image processing in porous media research. Adv. Water Resour. 31(9), 1174-1187 (2008). https://doi.org/10.1016/j.advwatres.2008.01.022 
Krevor, S., Blunt, M.J., Benson, S.M., Pentland, C.H., Reynolds, C., Al-menhali, A., Niu, B.: Capillary trapping for geologic carbon dioxide storage-from pore scale physics to field scale implications. Int. J. Greenhouse Gas Control 40, 221-237 (2015). https://doi.org/10.1016/j.ijggc.2015.04.006

Lai, P., Moulton, K., Krevor, S.: Pore-scale heterogeneity in the mineral distribution and reactive surface area of porous rocks. Chem. Geol. 411, 260-273 (2015). https://doi.org/10.1016/j.chemgeo.2015.07.010

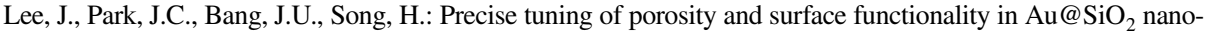
reactors for high catalytic efficiency. Chem. Mater. 20(18), 5839-5844 (2008). https://doi.org/10.1021/ $\mathrm{cm} 801149 \mathrm{w}$

Leu, L., Berg, S., Enzmann, F., Armstrong, R.T., Kersten, M.: Fast X-ray micro-tomography of multiphase flow in Berea sandstone: a sensitivity study on image processing. Transp. Porous Media 105(2), 451-469 (2014). https://doi.org/10.1007/s11242-014-0378-4

Lin, Q., Bijeljic, B., Pini, R., Blunt, M.J., Krevor, S.: Imaging and measurement of pore-scale interfacial curvature to determine capillary pressure simultaneously with relative permeability. Water Resour. Res. 54(9), 7046-7060 (2018). https://doi.org/10.1029/2018WR023214

Newman, J.: Optimization of porosity and thickness of a battery electrode by means of a reaction-zone model. J. Electrochem. Soc. 142(1), 97-101 (1995)

Peksa, A.E., Wolf, K.H.A., Zitha, P.L.: Bentheimer sandstone revisited for experimental purposes. Mar. Pet. Geol. 67, 701-719 (2015). https://doi.org/10.1016/j.marpetgeo.2015.06.001

Raeini, A.Q., Blunt, M.J., Bijeljic, B.: Modelling two-phase flow in porous media at the pore scale using the volume-of-fluid method. J. Comput. Phys. 231(17), 5653-5668 (2012). https://doi.org/10.1016/j. jcp.2012.04.011

Raeini, A.Q., Bijeljic, B., Blunt, M.J.: Generalized network modeling: network extraction as a coarse-scale discretization of the void space of porous media. Phys. Rev. E 96(1), 1-17 (2017). https://doi.org/10.1103/ PhysRevE.96.013312

Saxena, N., Hofmann, R., Alpak, F.O., Dietderich, J., Hunter, S., Day-stirrat, R.J.: Effect of image segmentation \& voxel size on micro-CT computed effective transport \& elastic properties. Mar. Pet. Geol. 86, 972-990 (2017). https://doi.org/10.1016/j.marpetgeo.2017.07.004

Scanziani, A., Singh, K., Blunt, M.J., Guadagnini, A.: Automatic method for estimation of in situ effective contact angle from X-ray micro tomography images of two-phase flow in porous media. J. Colloid Interface Sci. 496, 51-59 (2017). https://doi.org/10.1016/j.jcis.2017.02.005

Scanziani, A., Singh, K., Bultreys, T., Bijeljic, B., Blunt, M.J.: In situ characterization of immiscible three-phase flow at the pore scale for a water-wet carbonate rock. Adv. Water Resour. 121, 446-455 (2018). https://doi. org/10.1016/j.advwatres.2018.09.010

Schindelin, J., Arganda-Carreras, I., Frise, E., Kaynig, V., Longair, M., Pietzsch, T., Preibisch, S., Rueden, C., Saalfeld, S., Schmid, B., Tinevez, J.Y., White, D.J., Hartenstein, V., Eliceiri, K., Tomancak, P., Cardona, A.: Fiji: an open-source platform for biological-image analysis. Nat. Methods 9(7), 676-682 (2012). https ://doi.org/10.1038/nmeth.2019

Schlüter, S., Sheppard, A., Brown, K., Wildenschild, D.: Image processing of multiphase images obtained via X-ray microtomography: a review. Water Resour. Res. 50(4), 3615-3639 (2014). https://doi. org/10.1002/2014WR015256

Sikha, G., Popov, B.N., White, R.E.: Effect of porosity on the capacity fade of a lithium-ion battery. J. Electrochem. Soc. 10(1149/1), 1759972 (2004)

Singh, K., Blunt, M.: High resolution X-ray micro-tomography datasets for in-situ effective contact angle analysis in carbonate rocks (2018). https://doi.org/10.17612/P7D95F. http://www.digitalrocksportal.org/proje cts/125. Accessed 1 Feb 2018

Singh, K., Bijeljic, B., Blunt, M.: Imaging of oil layers, curvature, and contact angle in a mixed-wet and a waterwet carbonate rock. Water Resour. Res. 52, 1716-1728 (2016). https://doi.org/10.1002/2015WR018072

Sok, R., Varslot, T., Ghous, A., Latham, S., Sheppard, P., Knackstedt M.A.: Pore scale characterization of carbonates at multiple scales: integration of micro-CT, BSEM, and FIBSEM. Petrophysics 51, 379-387 (2010)

Soulaine, C., Gjetvaj, F., Garing, C., Roman, S., Russian, A., Gouze, P., Tchelepi, H.A.: The impact of subresolution porosity of X-ray microtomography images on the permeability. Transp. Porous Media (2016). https://doi.org/10.1007/s11242-016-0690-2

Supek, F.: Fastrandomforest (2008). https://code.google.com/archive/p/fast-random-forest/. Accessed 1 Jan 2018

Wildenschild, D., Sheppard, A.P.: X-ray imaging and analysis techniques for quantifying pore-scale structure and processes in subsurface porous medium systems. Adv. Water Resour. 51, 217-246 (2013). https://doi. org/10.1016/j.advwatres.2012.07.018 
Wu, C.D., Hu, A., Zhang, L., Lin, W.: A homochiral porous metal-organic framework for highly enantioselective heterogeneous asymmetric catalysis. J. Am. Chem. Soc. 127(25), 8940-8941 (2005). https://doi. org/10.1021/ja052431t

Publisher's Note Springer Nature remains neutral with regard to jurisdictional claims in published maps and institutional affiliations. 